\title{
The doctor is in - your workplace
}

$\mathrm{N}$ eed to see a doctor? For an increasing number of American employees, the solution is to stroll down the hallway.

It seems the dusty occupational health clinic, complete with the crusty staff nurse, is evolving into a full-service primary care facility, particularly for Fortune 500 companies looking to shave costs associated with medical insurance plans.

"What's different today is the former occupational health centres have been expanded to provide services that would be considered more like primary care," including diagnostic screening and testing, as well as urgent care, says Helen Darling, CEO and president of the Washington, DC-based National Business Group on Health.

The office clinics are typically staffed by physicians, registered nurses, laboratory technicians and case managers for workers' compensation, says Stuart Clark, president of commercial services for Reston, Virginia-based Comprehensive Health Services, which operates over 100 onsite health centres across several states.

Large corporations are responsible "dollar-for-dollar for the entire cost of health care for their covered population," says Clark. "So if the population is older and unhealthy, then the employer will have very expensive health care costs versus an employer that has a younger population and one that is healthier."

Many physicians find the model appealing because they get to work regular business hours, don't have to do the unpaid administrative work, such as billing, and don't have to deal with the stress of medical malpractice as the company covers liability.

Clark adds that the quality of the medical services provided in onsite clinics is high as Comprehensive Health Services insists that doctors utilize upto-date clinical practice guidelines and protocols. It provides 300 sets of guidelines and protocols to all clinics.

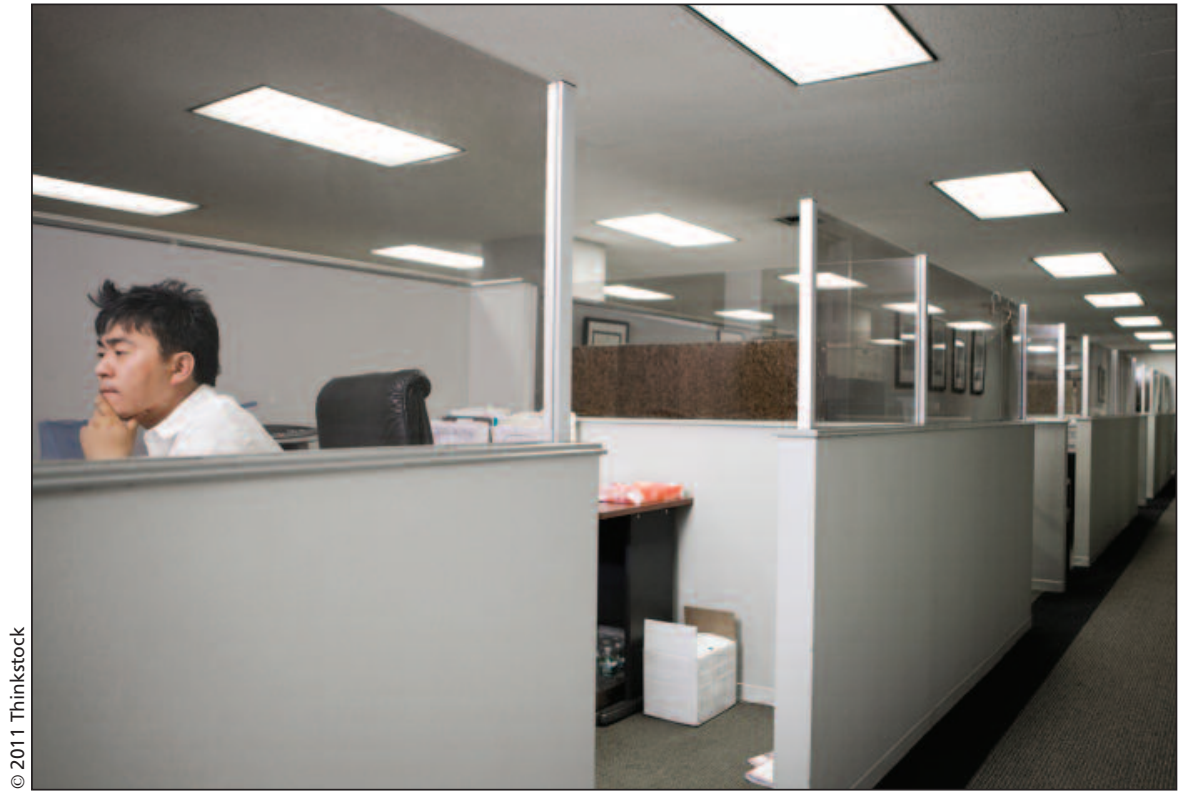

For an increasing number of office workers in the United States, a visit to the doctor requires only a stroll down the hallway.

"Our emphasis is on quality and the only way to achieve quality in health care is to help support the physicians in use of evidence-based medicine guidelines," says Clark adding that doctors appreciate the requirement as they aren't obliged to personally ensure that they are using the most recent guidelines. Instead of physicians having to do their own research, "we simply provide embedded decision support tools in our technology that ensures that they are following algorithms that keep them compliant with evidence-based guidelines," Clark adds. "Whether you're Canadian or American, that's of interest if you're a doctor."

Employers find the onsite clinic approach as appealing because they save money over the long-term, Clark says. Over the course of three to four years, a company can generally recover the cost of building a health facility, as well as recover operating costs at a $\$ 2.5$ to $\$ 1$ ratio because of improved productivity and reduced absenteeism, he says. The firm's website reports that one client saved $\$ 1.4$ million in six months (www.chsmedical.com/ServOn SiteHealth.html).
The savings accrue in part because employees don't need to take off work to travel to their doctor's office, which is often near their home. "In this country, many people in cities have very long commutes [to work] so you basically end up taking the day off," Darling says, adding that companies "don't lose the individual's time which is costly."

Onsite health centres can also have direct benefits for employees, such as reduced or waived copayments for medical services or drugs, Clark says, In some cases, employees have even made the centres available to the families of employees.

Employees "love the convenience and accessibility and it also makes them feel like the employer cares about them," Darling says.

Translating the model to Canada would be problematic as essential medical services are covered by provincial medicare plans, so the benefits would be strictly confined to productivity gains, Clark says. "Without the hard dollar savings to point to the employer, it is very difficult, in our opinion, to make it work up there." 
When Comprehensive Health Services was examining the possibility of expanding into Canada, it also concluded that doing so might be problematic because it could compromise the Canada Health Act, Clark adds. If an "employer opens an onsite health centre for its employees, it has to be open to the general public."

But the issue is not as clear cut as that.

"Companies may have occupational health clinics that are available to their employees," Ontario Ministry of Health and Long-term Care spokesperson Andrew Morrison writes in an email. "However, no payment can be made by an individual for preferred access to insured services."
A clinic does not have to be open to all patients, Morrison writes. The province will cover all insured services performed by health care providers so long as eligibility terms and conditions are met.

But while onsite primary health centres have not taken traction in Canada, there are signs that "workplace wellness" programs are on the rise, and some provinces, such as Alberta, even have programs designed to encourage employers to adopt such initiatives.

"Research has suggested that businesses who invest in workplace wellness programs see a return on investment \$3 for every dollar invested. These savings are witnessed through lowered healthcare costs, decreased absenteeism and decreased workers' compensation claims," an Alberta Ministry of Health and Wellness spokesperson writes in an email.

There's also a "niche" market for office clinics covering nonessential medical services, such as chiropractic and physical therapy, says James Beaumariage, senior vice president for pharmacy services for the drug retailer Rexall. Such clinics would be of value to firms who have large numbers of employees with repetitive stress injuries and back problems, Beaumariage says. - Erin Walkinshaw, Ottawa, Ont.

CMAJ 2011. DOI:10.1503/cmaj.109-3931 